\title{
Procedimento Administrativo Padrão: necessidade de elaboração e normatização no âmbito da Polícia Militar do Tocantins
}

Standard Administrative Procedure: need for elaboration and regulation within the scope of the Military Police of Tocantins

Procedimiento Administrativo Estándar: necesidad de elaboración y regulación en el ámbito de la Policía Militar de Tocantins

Recebido: 16/08/2021 | Revisado: 23/08/2021 | Aceito: 24/08/2021 | Publicado: 26/08/2021

\author{
Philipe Lira de Carvalho \\ ORCID: https://orcid.org/0000-0002-4778-4274 \\ Polícia Militar do Estado do Tocantins, Brasil \\ E-mail: lirapmto@gmail.com \\ Frank Cynatra Sousa Melo \\ ORCID: https://orcid.org/0000-0001-8824-6811 \\ Polícia Militar do Estado do Tocantins, Brasil \\ E-mail: frankcynatra@gmail.com \\ Marcos Antônio Negreiro Dias \\ ORCID: https://orcid.org/0000-0003-1964-620X \\ Polícia Militar do Estado do Tocantins, Brasil \\ E-mail: marcosnegreiros1985@gmail.com \\ José Carlos da Costa Abreu \\ ORCID: https://orcid.org/0000-0002-0332-8402 \\ Polícia Militar do Estado do Tocantins, Brasil \\ E-mail: cad.abreu@hotmail.com \\ Messias Rogério Araújo Albernaz \\ ORCID: https://orcid.org/0000-0003-2101-1166 \\ Polícia Militar do Estado do Tocantins, Brasil \\ E-mail: messiasrogerio.albernaz@gmail.com
}

\begin{abstract}
Resumo
Este artigo teve como finalidade mostrar a importância de confecção e normatização na esfera da Polícia Militar do Estado do Tocantins do Procedimento Administrativo Padrão (PAP). O trabalho em tela quando colocado em prática vai proporcionar padronização aos policiais militares tocantinenses que atuam no serviço administrativo. Além disso, se houver a implementação dessa proposta, vai refletir diretamente na excelência do trabalho prestado à população tocantinense. Dessa forma, esse trabalho científico chegou à conclusão que a PMTO necessita padronizar seus procedimentos administrativos. Na parte metodológica, a pesquisa é descritiva, bibliográfica, documental, exploratória e de natureza aplicada, realizando uma abordagem de cunho qualitativo qualitativa e empregando-se o método dedutivo. Enfim, com a normatização da supracitada proposta, a sociedade terá um serviço prestado de melhor qualidade e os integrantes do serviço administrativo da PMTO potencializarão a produtividade e consequentemente a instituição se fortalecerá ainda mais ao elaborar e implantar essa padronização de atividades administrativas.
\end{abstract}

Palavras-chave: Polícia Militar; Tocantins; Normatização; Procedimento administrativo padrão.

\begin{abstract}
This article aimed to show the importance of preparation and regulation in the sphere of the Military Police of the State of Tocantins of the Standard Administrative Procedure (PAP). The work on screen, when put into practice, will provide standardization for the Tocantins' military police who work in the administrative service. Furthermore, if this proposal is implemented, it will directly reflect on the excellence of the work provided to the population of Tocantins. Thus, this scientific work reached the conclusion that the PMTO needs to standardize its administrative procedures. In the methodological part, the research is descriptive, bibliographical, documental, exploratory and of an applied nature, carrying out a qualitative qualitative approach and using the deductive method. Finally, with the regulation of the aforementioned proposal, the company will have a better quality service provided and the members of the PMTO administrative service will boost productivity and, consequently, the institution will be further strengthened by preparing and implementing this standardization of administrative activities.
\end{abstract}

Keywords: Military Police; Tocantins; Standardization; Standard administrative procedure. 


\section{Resumen}

Este artículo tuvo como objetivo mostrar la importancia de la preparación y regulación en el ámbito de la Policía Militar del Estado de Tocantins del Procedimiento Administrativo Estándar (PAP). El trabajo en pantalla, cuando se ponga en práctica, proporcionará una estandarización para la policía militar de Tocantins que trabaja en el servicio administrativo. Además, de implementarse esta propuesta, se reflejará directamente en la excelencia del trabajo brindado a la población de Tocantins. Así, este trabajo científico llegó a la conclusión de que la PMTO necesita estandarizar sus procedimientos administrativos. En la parte metodológica, la investigación es descriptiva, bibliográfica, documental, exploratoria y de carácter aplicado, realizando un abordaje cualitativo cualitativo y utilizando el método deductivo. Finalmente, con la reglamentación de la propuesta mencionada, la empresa contará con un servicio de mejor calidad brindado y los integrantes del servicio administrativo de la PMTO impulsarán la productividad y, en consecuencia, la institución se fortalecerá aún más mediante la preparación e implementación de esta estandarización de actividades administrativas.

Palabras clave: Polícia Militar; Tocantins; Estandarización; Procedimiento administrativo estándar.

\section{Introdução}

Dentre as forças de Segurança Pública em nível estadual, está a Polícia Militar que tem um importante papel de exercer o policiamento ostensivo e preservar a ordem pública, conforme está previsto na Carta Magna (Brasil, 1988).

Nesse diapasão, a Polícia Militar do Estado do Tocantins (PMTO) tem a missão precípua de realizar o policiamento ostensivo/preventivo/repressivo para garantia da lei e ordem pública em todo Estado tocantinense. No entanto, a PMTO não se resume apenas a essa atividade-fim (operacional), uma vez que compreende também a atividade-meio (administrativa), na qual são estabelecidos seus atos administrativos que implicam no âmbito operacional e que precisam ser padronizados para evitar disparidades entre os documentos, além de agilizar sua confecção.

Diante disso, a padronização, enquanto importante ferramenta para as instituições, propiciará qualidade do produto, além de maior eficiência, eficácia e celeridade nos atos da Administração Pública.

Assim, com as novas necessidades de gestão e de aprimoramento profissional, a pesquisa justifica-se pela importância de ser elaborada e normatizada a padronização de um Procedimento Administrativo Padrão - PAP para ser seguido na esfera da PMTO, visando que seus integrantes, que laboram na atividade administrativa, conheçam os protocolos padronizados, e assim, potencializem suas atividades para atingirem a missão constitucional com excelência.

Ademais, este trabalho tem sua importância de cunho profissional, social e institucional, porquanto ao ser normatizado e instituído um procedimento administrativo padrão, a sociedade receberá um serviço de maior qualidade. O policial militar passará a conhecer o passo a passo (o proceder) de toda sua atividade administrativa e isso consequentemente vai agilizar e potencializar a realização de seu ofício e a Polícia Militar do Estado do Tocantins se fortalecerá ao elaborar e instituir essa proposta.

Logo, a problemática desse estudo originou-se da importância que a Polícia Militar do Estado do Tocantins precisa elaborar e normatizar para padronizar o procedimento administrativo em âmbito institucional.

De forma exemplificativas, foram elencados os seguintes Órgãos de Segurança Pública, que já instituíram o Procedimento Administrativo Padrão em seu âmbito, são eles: Polícia Militar do Mato Grosso, Polícia Militar de Goiás, Corpo de Bombeiros de Santa Catarina, Guarda Civil Metropolitana do Estado de São Paulo e Polícia Militar do Estado do Pará.

O objetivo geral deste trabalho foi apresentar o Procedimento Administrativo Padrão e sugerir que seja confeccionado e normatizado um instrumento de padronização na Polícia Militar do Estado do Tocantins.

Para alcançar esse objetivo geral, foram delineados os objetivos específicos a seguir:

$\checkmark \quad$ Conceituar o Procedimento Administrativo Padrão, conforme os ensinamentos de renomados autores;

$\checkmark \quad$ Discorrer sobre a relevância do Procedimento Administrativo Padrão na PMTO;

$\checkmark \quad$ Pesquisar e exemplificar alguns órgãos brasileiros na área de Segurança Pública que instituíram o Procedimento Administrativo Padrão; 
$\checkmark \quad$ Discutir os resultados alcançados com a pesquisa e estimular que seja realizado pela PMTO um Manual de Procedimento Administrativo Padrão em âmbito estadual.

Dessa forma, este artigo científico foi distribuído dessa maneira: 1) introdução; 2) aspectos metodológicos; 3) resultados, nesta seção foram tratados sobre os aspectos conceituais e importância do Procedimento Administrativo Padrão, bem como foram apresentados órgãos na área de Segurança Pública que instituíram o PAP; 5) discussão dos resultados encontrados na pesquisa e sugerido a normatização para padronização do procedimento administrativo para os policiais militares que atuam em funções administrativas na PMTO; e 6) considerações finais, seção que consta um apanhado geral da pesquisa.

\section{Metodologia}

O presente artigo científico teve em sua metodologia natureza aplicada, tendo em vista que proporcionou novidades sobre o assunto para que possa ser aplicado na Polícia Militar do Tocantins.

Concernente aos objetivos empregou-se o descritivo e exploratório, os quais segundo Fontana (2018) serviram para pormenorizar de forma descritiva as características de determinada população, além de explorar características prévias sobre assunto em específico. Diante disso, a pesquisa contemplou ensinamentos de renomados autores sobre o procedimento administrativo padrão e proporcionado maior contato com a temática.

Ademais, foi utilizado o método dedutivo, pois conforme Mazucato (2018) a pesquisa partiu de uma constatação geral para verificar assuntos particulares, a partir de assuntos amplos para se chegar no específico, a fim de se alcançar um resultado conclusivo.

Ademais, esta pesquisa foi elaborada através de procedimentos bibliográficos e documentais, nos quais segundo Fontana (2018) ficaram vinculados à leitura de livros e trabalhos científicos em geral, além do processar manuseio de documentos, ao se verificar manuais de Procedimentos Administrativos Padrão de outras Instituições da área de Segurança Pública.

Por fim, foi utilizada uma abordagem qualitativa no trabalho, uma vez que foram realizadas interpretações de assuntos literários e de normas nacionais atinentes a temática.

\section{Resultados}

Não diferente das demais polícias militares do País, a PMTO tem como papel Constitucional, exercer o policiamento ostensivo e preservar a ordem pública (Brasil, 1988). Além da missão de repressão à criminalidade.

A Administração Pública exterioriza sua vontade através da edição de atos administrativos. Esses atos muitas vezes dependem de inúmeros atos intermediários que seguem um rito ou procedimento estabelecido por atos normativos da própria Administração ou por lei (Rosa, 2017).

Nessa esteira, Vieira Filho (2019) salienta que a organização que não padroniza suas atividades diárias não é capaz de propiciar qualidade no seu produto e ainda, destaca que a organização tem direito de escolher a melhor forma para elaborar a padronização.

Conforme Zatarin, Silva e Piacente (2020, p. 5):

A padronização atua como uma importante ferramenta na identificação de problemas nos ambientes administrativos. Permite criar um fluxo de atividades, reduzindo as variações nos procedimentos, determinando as melhores práticas para promover a qualidade do serviço e permitindo o treinamento simples do pessoal, de forma que uma pessoa seja capaz de realizar mais de uma atividade/serviço, dando maior agilidade ao fluxo. 
Ademais, a padronização deixará os atos administrativos transparentes, uma vez que promoverá a localização e indexação dos documentos oficiais da Administração Pública, bem como propiciará segurança jurídica, celeridade e eficiência, impulsionando o alinhamento de entendimentos entre os diversos agentes públicos que atuam no mesmo processo (Rio Grande do Sul, 2019). Segundo Martins et al. (2021), a eficiência na administração pública reduz a morosidade dos processos, bem como de seus gastos.

A Polícia Militar do Estado de Mato Grosso (2016, p. 8) instituiu seu Manual de Procedimento Administrativo Padrão em relação a sua Diretoria de Ensino, Instrução e Pesquisa - DEIP, no qual está expresso que:

As muitas demandas do Setor impõem a necessidade de dinamizar as ações nele desenvolvidas, para tanto o presente Procedimento Administrativo Padrão tem por finalidade ser uma ferramenta apta a proporcionar maior dinamismo na realização das tarefas administrativas da DEIP, através de uma linguagem simples e objetiva sem, contudo, esgotar o assunto. Trata-se de uma ferramenta basilar, que carece de aperfeiçoamento contínuo.

Importante ressaltar que a Polícia Militar do Estado de Goiás (2021, p. 1), por meio da Portaria no 14.640, de 23 de abril de 2021, decidiu "aprovar o Procedimento Administrativo Padrão - PAP 01/2021, que tem por finalidade de estabelecer critérios para a coordenação, fiscalização e desenvolvimento da gestão do TCO/PM [Termo Circunstanciado de Ocorrência] em cada Unidade Policial Militar do Estado de Goiás, anexo a esta Portaria”.

De igual modo, o Corpo de Bombeiros Militar do Estado de Santa Catarina - CBMSC (2019) também instituiu e regulamentou seu Procedimento Administrativo Padrão - PAP, através da Portaria no 386, de 19 de setembro de 2019. Assim como, a Guarda Civil Metropolitana - GCM do Estado de São Paulo (2020), a qual aprovou, por meio da Portaria 56/GCM/2020, o seu PAP para disciplinar aspectos referentes as concessões de elogio.

A Polícia Militar do Estado do Pará - PMPA (2017, p. 3) aprovou o seu PAP, através da Diretriz nº 001/2017, a qual se originou:

Com a implementação e execução do Planejamento Estratégico da PMPA pelo Comando Geral da Instituição, objetivando o atendimento das iniciativas estratégicas construídas para o Plano Estratégico 2015/2025, onde prevê, dentre várias, a padronização de todos os procedimentos existentes na PMPA, buscando a melhoria no atendimento ao cidadão, por meio de técnicas e atividades que propiciem a adoção de fundamentos dos Direitos Humanos, atendendo aos requisitos primordiais da Administração Pública Gerencial.

Nesta senda, Cury (2016) exalta a relevância dos manuais administrativos, tratando-os como documentos elaborados, visando padronizar procedimentos que devem ser notados nas mais variadas atividades, inclusive, sendo, um eficaz instrumento de racionalização de métodos, bem como de aperfeiçoamento de comunicações e de integração de diversos sistemas e subsistemas de uma organização.

Na visão de Mendonça (2014, p. 64):

[...] os manuais são instrumentos gerenciais, de relativa duração, que apresentam instruções de caráter normativo agrupadas por um invólucro característico, de fácil identificação, classificado, codificado, provido de índice e organizado a fim de aumentar ao máximo o seu valor como obra de referência. Obviamente, a relativa duração dos documentos, inseridos nos manuais, não os tornam eternos e, assim, eles devem ser encarados como instrumentos executivo-normativos cuja utilidade para a organização reside na razão direta de sua flexibilidade e agilidade em se adaptar às mudanças processadas em quaisquer dos assuntos que os integram.

Já para Oliveira (2019), manual é qualquer conjunto de normas, procedimentos e documentos atinentes às atividades, funções, objetivos, políticas, orientações e instruções que devem ser seguidos e devidamente cumpridos pelas pessoas que compõem uma empresa. E para sua elaboração, é importante ocorrer de forma conjunta, considerando-se a multiplicidade de 
atribuições nos diversos setores institucionais, divulgando-se aspectos relacionados à estrutura organizacional e seus métodos, rotinas e procedimentos administrativos básicos.

Segundo Ballestero-Alvarez (2014), esse tipo de manual estabelece um conjunto de técnicas, processos e métodos para a performance de atividades futuras, além de serem guias de raciocínio e de sequências de forma cronológica de atos.

Consoante os ensinamentos de Santos et al. (2018, p. 107), os manuais são vantajosos, pois:

Para que os processos sejam geridos no setor público é necessário considerar os princípios constitucionais. As estatais são obrigadas a aplicar as leis em seus processos, atividades e atos. O princípio da eficiência trouxe a garantia ao cidadão, de que os serviços públicos precisam ser prestados com rapidez, qualidade, rendimento funcional etc. A partir deste ponto, a administração pública precisa buscar alternativas para fazer cumprir o princípio da eficiência na prestação dos serviços públicos.

Di Pietro (2016, p. 767) menciona que Procedimento Administrativo é "o conjunto de formalidades que devem ser observadas para a prática de certos atos administrativos; equivale a rito, a forma de proceder; o procedimento se desenvolve dentro de um processo administrativo".

Segundo os ensinamentos dos autores Pelegrini e Sanches (2018, p. 145), o "atual contexto das ciências militares vem se inovando com o passar dos anos, o uso de métodos operacionais padronizados, tem surtido efeito e promovido prerrogativas na segurança pública". Diante disso, os procedimentos instigam a padronização das ações de uma Corporação com ações de inteligência.

Conforme as lições de Campos (2014), quando se elabora um procedimento em todas as funções das atividades administrativas, ocorrem poucos erros. Dessa forma, ao seguir uma uniformização de como proceder no decorrer dos processos administrativos, as informações das instituições ficam fortalecidas, pois é a melhor maneira para manter suas atividades potencializadas.

É importante mencionar que após consultas nos acervos da Corporação, conforme pesquisa realizada e pela vivência prática do cotidiano, verificou-se que a Corporação Militar, objeto deste estudo, não possui um procedimento administrativo padronizado em suas rotinas administrativas.

Nesse diapasão, um Manual de Procedimento Administrativo Padrão é de suma relevância para a Polícia Militar do Tocantins, pois, sendo uma instituição de muitas tradições, deve buscar a excelência na prestação de seus serviços.

$\mathrm{O}$ supracitado instrumento visa dar garantias do cumprimento das demandas administrativas de forma padronizada e técnica aos militares estaduais que trabalham no serviço administrativo da instituição.

Ademais, o Procedimento Administrativo Padrão visa estabelecer procedimentos de rotinas para que as atividades desenvolvidas sejam padronizadas segundo orientação e treinamento específicos.

\section{Discussão}

A Administração Pública exterioriza sua vontade através da edição de atos normativos. Daí a importância de serem tratados sobre os aspectos conceituais sobre o procedimento administrativo padrão.

Além disso, constatou-se que os renomados autores mencionados neste trabalho, colaboraram com seus ensinamentos, ficando compreensível que a padronização favorece as ações dentro de uma entidade, associadas aos princípios da eficiência, eficácia e celeridade. Assim, uma instituição/organização que não padroniza suas atividades de rotina não poderá garantir a qualidade do que faz.

No decorrer do trabalho, foram apresentados alguns Órgãos da área de Segurança Pública que aderiram ao Procedimento Administrativo Padrão, seja em um ou vários setores administrativos. Assim, verificou-se a aplicação desse 
instituto na Polícia Militar do Mato Grosso, Polícia Militar de Goiás, Corpo de Bombeiros de Santa Catarina, Guarda Civil Metropolitana do Estado de São Paulo e Polícia Militar do Estado do Pará.

Após consultas nos acervos da PMTO, conforme pesquisa realizada no decorrer deste trabalho científico e pela vivência prática do cotidiano, observou-se ela não possui um procedimento administrativo padrão em suas rotinas administrativas.

Diante disso, vários autores relatam sobre a necessidade que seja confeccionado um Manual que possa ser utilizado para padronização das sistemáticas operacionais, incluindo a padronização dos procedimentos administrativos na esfera da PMTO. Pelegrini e Sanches (2018) discorrem sobre a importância de padronização na Corporação, no contexto atual das ciências militares, para que auxiliem na eficiência e eficácia dos atos administrativos na segurança pública.

Além disso, Campos (2014) destaca sobre a necessidade da uniformização para proceder nos precisos decisórios, tendo em vista que fortalece as informações das instituições/organizações, pois é a forma mais eficaz para manter suas atividades aceitáveis.

Dessa forma, comprovou-se que a implantação de um Manual de Procedimento Administrativo Padrão, é necessária, tendo em vista que uma vez implantado, facilitará os trâmites administrativos decorrentes de sua padronização, com vistas a qualidade no serviço a ser prestado do profissional para a Corporação e para população tocantinense.

\section{Considerações Finais}

Esta pesquisa fez um apanhado dos resultados elencados na pesquisa científica que tem como temática o Procedimento Administrativo Padrão. Ademais teve como objetivo sugerir que seja realizado pela PMTO um Manual de Procedimento Administrativo Padrão - PAP em âmbito estadual.

Para isso, foi conceituado o procedimento administrativo padrão conforme ensinamentos de renomados autores. Na sequência, foi comentado sobre a relevância do Procedimento Administrativo Padrão na PMTO. Em seguida, foram apresentados os resultados elencados na pesquisa, entre eles: exemplificação de algumas instituições de Segurança Pública que instituíram o PAP. Após foram realizados comentários a respeito da necessidade dessa padronização.

Com o intuito de solucionar a problemática, conclui-se que é imperiosa a confecção de um Manual de Procedimento Administrativo Padrão e de normatização que poderá ser realizada, através de Portaria do Excelentíssimo Senhor ComandanteGeral da PMTO em âmbito Institucional.

Em face dessa pesquisa técnico-científica, conclui-se que consolidado está o entendimento de que é importante a padronização do procedimento administrativo para uniformização das atividades administrativas desempenhadas pelos integrantes da instituição. Dessa forma, pelos resultados adquiridos neste estudo, tem-se como meta possibilitar que haja trabalhos futuros que valorizem a padronização de procedimentos administrativos para propiciar eficiência, eficácia e celeridade nos atos da Administração Pública.

\section{Referências}

Ballestero-Alvarez, M. E. (2014). Manual de organização, sistemas e métodos: abordagem teórica e prática da Engenharia da Informação. (6a ed.), Atlas. Brasil. (1988). Constituição da República Federativa do Brasil de 1988. Planalto Brasília. http://www.planalto.gov.br/ccivil_03/constituicao/constituicao.htm. Campos, V. F. (2014). Qualidade total na padronização. (2a ed.), Ed. INDG.

Corpo de Bombeiros Militar do Estado de Santa Catarina - CBMSC. (2019). Portaria $\mathrm{n}^{\mathrm{o}}$ 386, de 19 de setembro de 2019. Procedimento Administrativo Padrão. https://documentoscbmsc.cbm.sc.gov.br/uploads/0b3b9928c226f727680452316881e0d0.pdf.

Cury, A. (2016). Organização e métodos: uma visão holística. (9a ed.), Ed. Atlas.

Di Pietro, M. S. Z. (2016). Direito Administrativo. (29a ed.), Ed. Forense. 
Research, Society and Development, v. 10, n. 11, e158101119475, 2021

(CC BY 4.0) | ISSN 2525-3409 | DOI: http://dx.doi.org/10.33448/rsd-v10i11.19475

Fontana, F. (2018). Técnicas de Pesquisa. In T. Mazucato (Org.), Metodologia da Pesquisa e do Trabalho Científico (pp. 59-80). Ed. Funepe.

Guarda Civil Metropolitana - GCM do Estado de São Paulo. (2020). Portaria 56/GCM/2020. Aprova o Procedimento Administrativo Padrão da Guarda Civil Metropolitana, que especifica. http://legislacao.prefeitura.sp.gov.br/leis/portaria-secretaria-municipal-de-seguranca-urbana-smsu-gcm-56-de-16-de-novembrode-2020/detalhe.

Martins, E., Dias, C. A., Santos, C. M., Souza, M. C., \& Gomes, L. de S. (2021). Análise integrativa sobre o processo administrativo disciplinar e o princípio da eficiência. Research, Society and Development, 10(4), e29610414124. http://dx.doi.org/10.33448/rsd-v10i4.14124.

Mazucato, T. (2018). A elaboração do pré-projeto. In T. Mazucato (Org.), Metodologia da Pesquisa e do Trabalho Científico (pp. 40-46). Ed. Funepe.

Mendonça, R. R. S. de. (2014). Processos administrativos. (3a ed.), Departamento de Ciências da Administração / UFSC; CAPES:UAB.

Oliveira, D. P. R. de. (2019). Sistemas, organização e métodos: uma abordagem gerencial. (21a ed.), Ed. Atlas.

Pelegrini, A. de J. N., \& Sanches, C. P. (2018). Procedimento operacional padrão na PMGO: a importância de sua existência, adoção e adesão. REBESP. 11(1). https://doi.org/10.29377/rebesp.v11i1.340.

Polícia Militar do Estado de Goiás (2021). Portaria nº 14.640, de 23 de abril de 2021. Aprova o Procedimento Administrativo Padrão - PAP 01/2021, da Coordenação do TCO-PM (EME), conforme especifica. https://www.pm.go.gov.br/wp-content/uploads/2021/07/pap.pdf.

Polícia Militar do Estado de Mato Grosso. (2016). Manual de Procedimento Administrativo Padrão. Cuiabá-MT. http://www.pm.mt.gov.br /documents/2459523/5153878/MANUAL+DE+PROCEDIMENTO+ADMINISTRATIVO+PADR\%C3\%83O-DEIP-2016.pdf/3b3a6d9a-a1c9-4912-86d12e0628a0e905.

Polícia Militar do Estado do Pará. (2017). Diretriz nº 001/2017 - Estado Maior Geral PM7. Estabelece o modelo de Procedimento Operacional Padrão (POP) $e$ de Procedimento Administrativo Padrão (PAP), a forma de confecção destes documentos e o setor validador na PMPA. https://www.pm.pa.gov.br/phocadownload/userupload/userupload/BGs/2017/03\%20MAR\%C3\%870/2017_03_27-adit059.pdf.

Rio Grande do Sul. Secretaria da Casa Civil. Subchefia Jurídica. (2019). Manual de boas práticas e de padronização dos atos administrativos normativos e ordinatórios na administração pública estadual. / Coordenação M. P. Möllmann. Secretaria da Casa Civil. https://casacivil.rs.gov.br/upload/arquivos/201911/12113138-manual-de-boas-praticas-de-atos-administrativos.pdf .

Rosa, M. F. E. (2017). Direito Administrativo - Coleção sinopses jurídicas: 19. (9a ed.), Ed. Saraiva.

Santos, L. D, L. dos, Florêncio, M. N. da S., Escobar, M. A. R., \& Zambanini, M. E. (2018). A importância dos manuais na gestão dos processos de trabalho com base na percepção da gerência: um estudo de caso aplicado nos correios do interior sergipano. Proceeding of ISTI/SIMTEC. 9(1), 105-115. http://www.api.org.br/conferences/index.php/ISTI2018/ISTI2018/paper/viewFile/552/271.

Vieira Filho, G. (2019). Gestão da qualidade total: uma abordagem prática. (6a ed.), Ed. Alínea.

Zatarin, J. K., Silva, V. de C., \& Piacente, F. J. (2020). Análise da padronização do trabalho na área de certificação digital: um estudo de caso. Research, Society and Development, 9(10), e309108394. 10.33448/rsd-v9i10.8394. 